\title{
Data Model in PLM System to support Product Traceability
}

\author{
Dharmendra K. Mishra ${ }^{1}$, Aicha Sekhari, ${ }^{1}$ Sebastien Henry ${ }^{2}$, Yacine Ouzrout ${ }^{1}$ \\ ${ }^{1}$ University Lyon 2 Lumiere, DISP Laboratory, France \\ ${ }^{2}$ University Claude Bernard Lyon 1, DISP Laboratory,France \\ \{dharmendra-kumar.mishra, aicha.sekhari,yacine.ouzrout\}@univ-lyon2.fr
}

Sebastien.henry@univ-lyon1.fr

\begin{abstract}
The demand of oxygen gas in Nepal is increasing. The gas companies manufacture the gas and supply to various hospitals and glass companies across the country. All the information management is done in manual basis and there is no any tracking mechanism. During some emergency, the gas companies immediately supply the gas to some customers without any record. This results the loss of cylinders in the network. The gas supply chain is complex network as a gas company has many customers which in turn takes gas from various distributers. In this paper, a trace algorithm is developed to implement data model to trace the product using a use case study in Oxygen gas supply chain in Nepal. Each cylinder has certain life span, so PLM system is used to record the information related with cylinders to check the quality issue of cylinders. The concept of SaaS (Software as a Service) of Cloud Computing is used to keep the traceability system in the cloud. A user friendly framework is conceptualized which facilitates the easy access of required information.
\end{abstract}

Keywords: Traceability, PLM, SaaS, Data Model

\section{Introduction}

High customers demand and competition among the manufacturers require safe and timely delivery of product at customer's doorsteps. Increased awareness of customers towards high quality product draws keen interest in developing traceability system in product supply chain. Many countries have legislative requirement to have traceability system across the supply chain especially in food and medical sectors. But, the requirement of such system in other supply chain network cannot be ignored. Meeting production plans and cost, fighting with counterfeit threats, achieving product safety, meeting regulatory compliance and enhancing the product quality make the traceability system important [1]. Traceability system reduces the overall production cost of companies which is another reason of making it important tool. In this way, it is now well known that the supply chain actors have to implement their traceability system not only to meet the legislative obligation but also to enhance their overall production plan. Considerable researches are being done to 
trace the product at any point from the academia. The summary of these research works are explained in section 2 .

In Nepal, there is considerable number of manufacturers and distributers of Oxygen gas. Gas manufacturers refill the empty cylinders and despatch these to distributers as per the order. There exists no computerized system to trace the cylinders after these are despatched from the companies. The author visited the gas companies based on Kathmandu, the capital city of Nepal and asked the owner to know whether they are facing any problem in supply chain network. It is found that some of time they lose their cylinder in the market when it goes to customers as some customer do not return the empty cylinders and they have no idea of where their cylinder is. This results the companies to face ample amount of financial loss. There is chance of misuse of these cylinders resulting big threats to the patients and this also affect the company's brand. Product tracing in complex network is a challenge. There exists traceability systems in literature to cope with this challenge. Among them, many have proposed to use RFID tagging in the product that records the data associated with the product form its production till use. A web based application access these data.

The products have their lifecycle from conception to end. In case of Oxygen gas cylinders, the gas is consumed but the cylinders have limited life span. There are two categories of cylinders: Small and large. Small cylinders are used for personal purpose and the large ones are being used by hospitals. Lifespan of small cylinders is up to 12 years and for the larger ones it ranges from 17 to 20 years. Lifespan also depends on how the cylinders are handled by the persons who fill, transport, and store and use it. There is chances of rust formation when cylinders come to contact with air during refilling. This affects the gas quality. A PLM system will be used to manage and store the cylinder's refilling information throughout its life cycle to check the quality issues by connecting the PLM system with traceability system. The purpose of this work is to implement a traceability data model considering the use case of oxygen gas supply chain. In previous work by these authors, a bi-graph model was proposed to trace the product in global supply chain based on which a data model is presented [2]. In this work, model is enhanced by developing trace algorithm and a trace framework is proposed by connecting the traceability system with PLM system.

\subsection{Traceability Data Model}

Some researcher works in the field of traceability data model. Thierno et al develop a data model to handle unitary traceability based on IEC 62264 and GS1 global standard [15]. The author aims to represent the internal traceability data to find the root cause and facilitate data exchange [2]. A data model is proposed to support the traceability process in the food supply chain [16]. Similarly, M. khabbaji et al propose data model to manage the lot traceability on the basis of make order [17]. The model is said to support to control the material flow in all quality and production 
process. Some other works $[17,18]$, develop internal traceability data model in terms of materials and process data registration. We propose global traceability data model tracing each data at each point from production till the use of the product [2]. There is many to many relationship between the actors sharing the data. The data generated at each point of the supply chain are company name, product s.n, date of order received, product received information, distributer id, order delivery report etc. It is mandatory for the actors to enter all the necessary information in the system.

\subsection{Oxygen Gas Supply Chain Nepal}

In this work, the authors implements the supply chain process of Sagarmatha Oxygen Gas Company based on Kathmandu, Nepal. This Company manufactures Oxygen and Nitrogen gas and supplies to various hospitals and glass industries through various distributers throughout Nepal. Authors consider oxygen gas network only as a case study to implement the trace algorithm. It has around 27 hospitals and 200 glass product manufacturers as customers. Hospital use the oxygen gas for patient's treatments and the glass manufacturers use it to cut the glass. The gas company has 23 distributers through which it supplies the product to its various customers. Considering the use of Oxygen in the hospitals, the company undergoes through continuous manufacturing process. It does not wait for order from the customers to satisfy timely delivery of gas cylinders. It is found that the company pay proper attention in quality checking process. When the gas is manufactured a continuous quality checking process is adopted by purity operator before refilling the cylinders. The company has many distributers which supplies the gas cylinder to customers, in turn, the customers have also many suppliers of the gas cylinder. As shown in the figure 1 the gas company manufactures and supplies the gas upon receipt of order from its distributers. Distributers receives order from the customers and supplies to them. All the order management process is done manually.

Though it is mentioned in the previous paragraph there are 27 distributers and more than 200 customers the company has, authors took only three to show the representations. In the next section, the overall of tracing system is described.

\subsection{Traceability System}

Enhancing the product quality, delivering the product on right time in the market, fighting product counterfeiting to protect the brand are the key challenges companies must have to cope with [2]. A proper trace and track mechanism is quite helpful and essential to cope with these challenges. There are many actors in supply chain scattered around the globe having multiple roles and objectives, make the modern day supply chain: a complex system [2]. Tracing all the information at each point is still a challenge. Many works are found in literature in this area. The concept of 
traceability first used by the field related with space, health and military [3]. Growing competitive environment, consumer's awareness about the product usage, customer's safety from the contaminated product and legislative requirement cause manufacturers to have their product traceability system in last 2 decades.

There are two terms "tracking" and "tracing" mostly used in traceability literature. Tracking is the process of going forward in upstream to follow the product while tracing is going backward in downstream to find the origin of the product. Product's visibility across the entire supply chain network is essential which is achieved by these two process. A good traceability system must have to implement these two process efficiently [1].

The traceability system consists of identifying the product location, storing the data, capturing the products related information and disseminating the information across the global network. Use of RFID is common tool being used in product identification using some standards. GS1 is a global standard organization very popular among the general traceability standards. Apart from RFID and GS1 global standards there have been some common techniques being used to implement the traceability system. GPS (Global positioning System), use of Cloud model and bar codes are to name few among them.

\section{Research Problem}

Many works have been found in the traceability literature that includes the development of a traceability model or a framework towards tracing the product's information in supply chain. In 2007, A. Reggattieri et. al studied the need and importance of traceability in food supply chain performing a case study of traceability solution used by Parmigiano Reggiano cheese, a famous Italian Cheese [5]. The Italian Pizza uses the RFID tag in the whole cheese and alphanumeric code in the partitioned one. The RFID tag and the code store all the related information from processing of bovine milk in dairy to making of cheese to warehousing and packaging. The traced data are stored in a central server from where they can be accessible using a dedicated website by entering the alphanumeric code. In their work " a secure RFID-based Track and Trace solution in Supply Chains" in 2008 W. He et. al proposed use of RFID based on EPC global standard to identify products in the whole supply chain [6]. The important aspect of this work is that the authors proposed two levels of trust model for data security. Any web based services can be used to trace the data from the EPCIS server through this two level of trust model. In 2009 W.He, N. Jhang et.al studied the use of RFID, GPS and EPC to trace the products [7]. The authors implemented an integrated model which contains RFID tag, EPC global standard and GPS receiver. A web based application can be used to access the information from the central server. Use of RFID, EPCIS and GIS has been studied in 2010 by R. Shougang. Here the authors proposed that every meat 
product have RFID tag with EPC on it. At each point of supply chain the information are updated by EPCIS system that are integrated with corresponding geographic information of each distribution point and users get these data by inputting the EPC on given traceability system [8]. A cloud platform for the traceability was proposed in 2011 by the authors R. Z. Cao, X. Y. He et al [9].

Next, in 2012, a service oriented Livestock management system using mobile cloud architecture was proposed by C. Teng, K. Brown et. al [10]. In this work, the RFID tag is attached in the ear of the animal. Microsoft azure and service oriented architecture is used for the information management. The mobile client application interfaces with the RFID reader to get the unique ID from the tag. Farmers use web based application to access the information. Similarly, an innovative cloud based traceability architecture and service was proposed by N. Madhoun, F. Amine et. al in the same year [11].

Use of wireless sensor networks (WSN) was studied by I. Exposito, I. Cuinas et. al in 2013 to develop traceability solution for wine production company [12]. The authors proposed to use RFID which holds all the required information from grapes harvesting to bottling. Sensors are put on six different points across the wine production lines to measure ambient and soil temperature and humidity and leaf wetness. The information are stored on the central server and accessed by a customized desktop application through API. In another work by A. Kassahun, R. Hartog et. al in 2014 proposed to keep EPCIS on the cloud to maintain transparency in meat supply chain [13].

A case study method is adopted to develop a traceability framework to maintain transparency and to achieve security in dairy supply chain network in India [19]. Event driven process chains (EPCs), E-R model and activity based costing (ABC) are used to develop a framework that define and analyse the current supply chain [20]. Authors develop business process reengineering for fourth range vegetable product supply chain and set up computerized system to manage product traceability. A multifunctional database model is developed to manage internal traceability of grain elevator [21]. The model stores product and quality information related to individual grain lots. A UML model to develop and implement internal traceability system of vegetable supply chain is designed [22]. Authors develop UML class diagram of traceability data model. RFID is considered to identify the products information at every point. GS1 standard has been used for web based implementation of the model to manage internal as well as chain traceability.

The conclusion of all the above works is to propose a traceability system with basic information. There is lack of common standards between the actors which result loss of information. No works explain the need of PLM system with the traceability system. As the every product has its life span, the products information from its conception to use in the PLM system helps proper data management. Interfacing the PLM system with the traceability system will add the extra value in the traceability literature. 
This work solves the issue by implementing the traceability data model using a case study.

\section{Implementation}

\subsection{Traceability System Requirements}

A proper traceability system must be able to store and communicate with actors about product's location, quality, user's safety, actors and process involved in product's manufacturing, handling, transportation and storage [4]. To achieve this, the identification of usage requirements is the first task to be accomplished. UML use case diagram is used to identify the traceability system requirements in this work. Fig. 1 below shows the diagram.

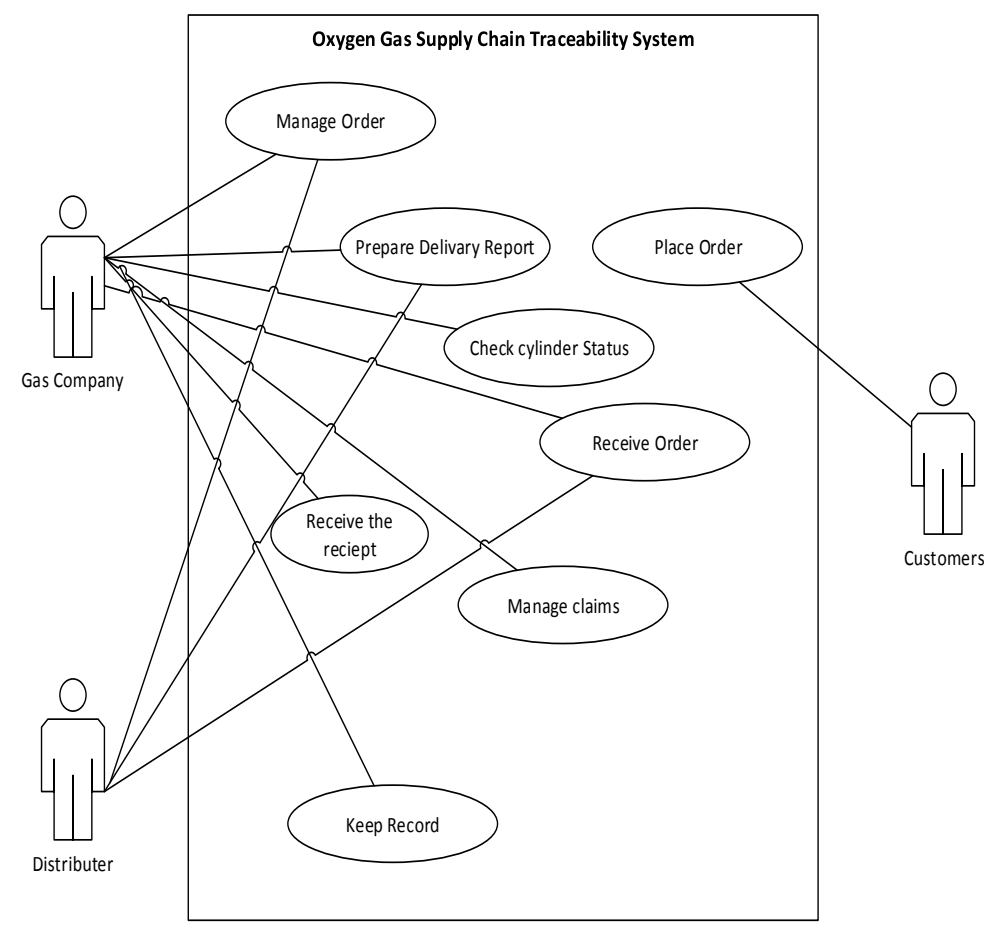

Fig. 1. Use Case Diagram of Oxygen Gas Supply Chain Traceability System

As shown in the diagram, the system has following use cases associated with each actors:

- Mange Order: Distributer receives order form the customer and accordingly it places the order to the gas company. Management of order includes date and time of orders received and placed, quantity of gas cylinder demanded and delivery order given to the production team. 
- Prepare Delivery report: The production team prepares delivery report for the distributer when the cylinders are ready for despatch. Accordingly, distributers prepare delivery report for the customers as per their order.

- Check Cylinder status: Gas Company checks the status of cylinder which includes quality testing and location identification of the cylinder. The cylinder has been given unique identification as per GS1 standard, GTIN. GTIN is a 14 digit (EAN.UCC) data structure which is encoded in various type of carrier. In this work, RFID tagging is used on each cylinder. This uniquely identify the cylinder at each point of gas supply chain.

- Receive receipt: When the gas cylinders are delivered to either distributer or the customers each actors must authenticate that the cylinders are properly received as per their order.

- Place Order: Customers place order to the distributers as per their requirements and distributers place order to the gas company.

- Receive Order: Gas Company receive order from Distributers and Distributers receive order from Customers.

- Keep record: Each actor keeps record of the date of placing or receiving order, date and no. of cylinders despatched and received. The gas company despatches the filled cylinders after receiving the empty cylinders only. So, gas company must keep record whether it receives the empty cylinders from the distributer or not. Similarly, distributer also note the acceptance of such cylinders from customers.

- Manage Claims: The system users manage claims as per the data stored in the system. For example, if a cylinder is lost after being despatched, the users must know where it has gone. 


\subsection{OGtrace Algorithm}

The main problem the gas company faces is the loss of empty cylinder sometimes. The cylinders given to some customers are not returned and the company does not have proper information to which customers the cylinders have.

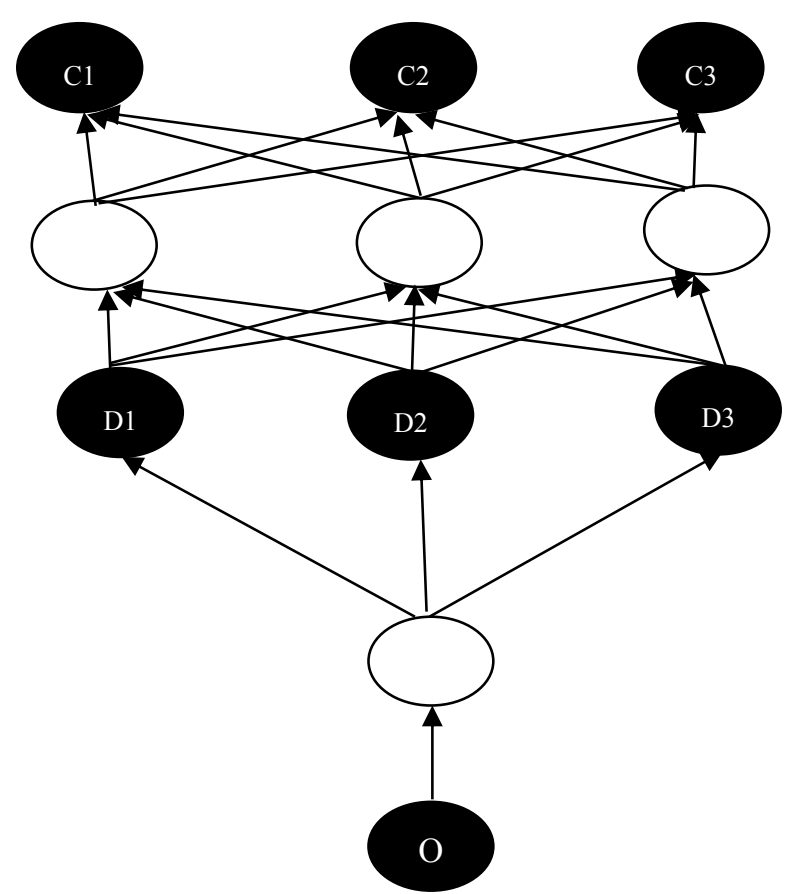

Fig. 2. bi-graph model of oxygen gas supply chain

To overcome this problem we have developed our OGCtrace (Oxygen Gas Cylinder trace) algorithm using bi-graph model.

In graph theory, bi-graph (Short form of bipartite graph) is a graph, whose vertices are divided into two disjoint independent sets $\mathrm{U}$ and $\mathrm{V}$ of which there is an edge from every vertices in $U$ to that of in $V$, such that $U U V=$ All the vertices in the set and $\mathrm{U} \cap \mathrm{V}=\varphi$ that is null [13].

Figure 2 shoes the bi-graph model of gas supply chain. In the model, the actors belong to one set of vertices while the cylinders (Empty or Filled) to other. The filled circles in the figure 3 shows the actors and other circle denotes either filled or empty cylinders. O denotes the oxygen gas company, D is for the distributer and $\mathrm{C}$ is for the customer. We model the graph as $G(A, B, E)$ where A represents the actors (Gas Company, Distributers and Customers), B represents filled or empty cylinders and $\mathrm{E}$ represents the edge connects one vertex from other.

Further we assign the weight to the edges as follow: 


$$
\mathrm{W}(\mathrm{E})=\left\{\begin{array}{l}
=0, \text { if } B \rightarrow A, \text { if } B \rightarrow A \\
=1, \text { if } A \rightarrow B, \text { if } A \rightarrow B
\end{array}\right.
$$

Since the nodes are different in 2 sets, the differences are shown as follow [14]: Let $\phi(A)$ be a function representing the various actors then

$$
\phi(A)=\left\{\begin{array}{l}
\theta 1, \text { ifAisGasCompany } \\
\theta 2, \text { ifAisDistributer } \\
\theta 3, \text { ifAisCustomer }
\end{array}\right.
$$

And let $\varphi(\mathrm{B})$ be the function representing cylinders then we represent it as follow:

$$
\varphi(B)=\left\{\begin{array}{l}
\lambda 1, \text { ifBisemptycylinder } \\
\lambda 2, \text { ifBisfilledcylinder }
\end{array}\right.
$$

Now we model our graph as $\mathrm{G}(\mathrm{A}, \phi(A), \mathrm{B}, \varphi(\mathrm{B}), \mathrm{E}, \mathrm{W}(\mathrm{E}))$. Based on these parameters the OGCtrace algorithm below trace a lost cylinder at any point in the supply chain. We start from a vertex in the graph and start searching the graph using breadth first search algorithm. Given below is the pseudocode of the algorithm.

1. Set a vertex ' $\mathrm{V}$ ' as a point of interest in $\mathrm{SC}$;

2. Initialize the graph $\mathrm{G}(\mathrm{A}, \phi(\mathrm{A}), \mathrm{B}, \varphi(\mathrm{B}), \mathrm{E}, \mathrm{W}(\mathrm{E}))$;

3. Initialize the traced_data $=0$;

4. Add ' $\mathrm{V}$ ' to queue;

5. While Queue is not empty

Delete $\mathrm{V}$ from queue;

Let ' $u$ ' be a vertex adjacent to $\mathrm{V}$;

While u\{

Add u to queue;

Store the information in traced_data;

Update $\mathrm{G}()$;

\}

$\mathrm{U}=$ next vertex that is adjacent from $\mathrm{V}$;

Return traced_data;

Return G();

The algorithm is based on queue data structure, in which the nodes are being added in the queue until a faulty node is found. The faulty node is a point in supply chain 
where a problem is encountered. The traced_data in algorithm, has the information of lost cylinder. Upon receiving the location of the cylinder, the gas manufacturer checks the quality issue of the cylinder.

\subsection{Proposed Oxygen Gas Traceability System (OGTS) framework}

The gas company imports the cylinder from other countries. There is no company that manufactures the oxygen gas cylinder in Nepal.
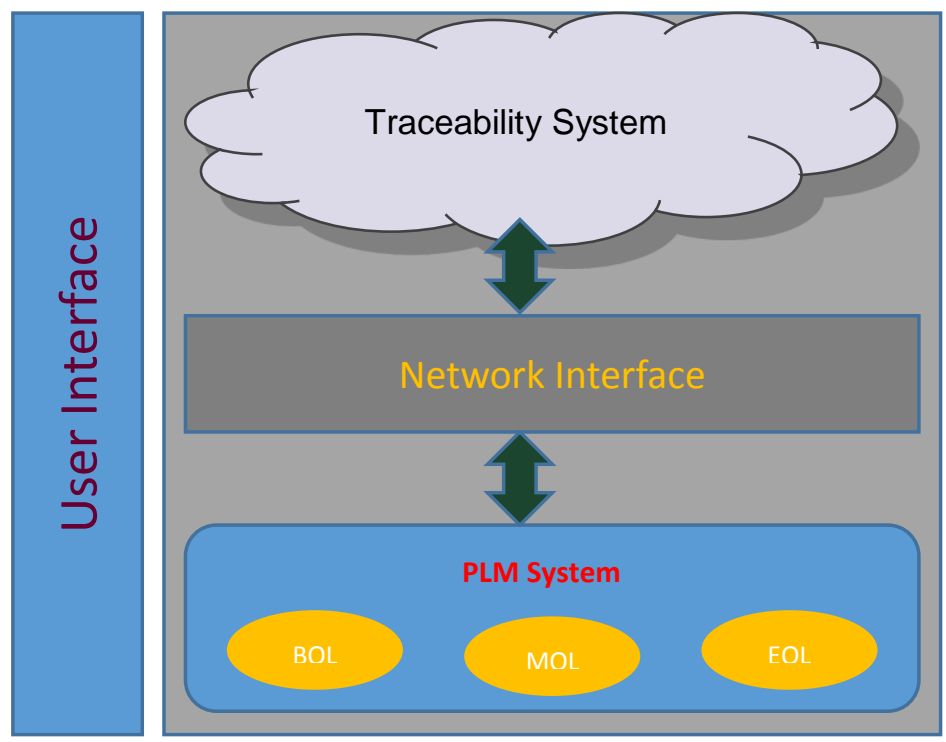

Network Interface
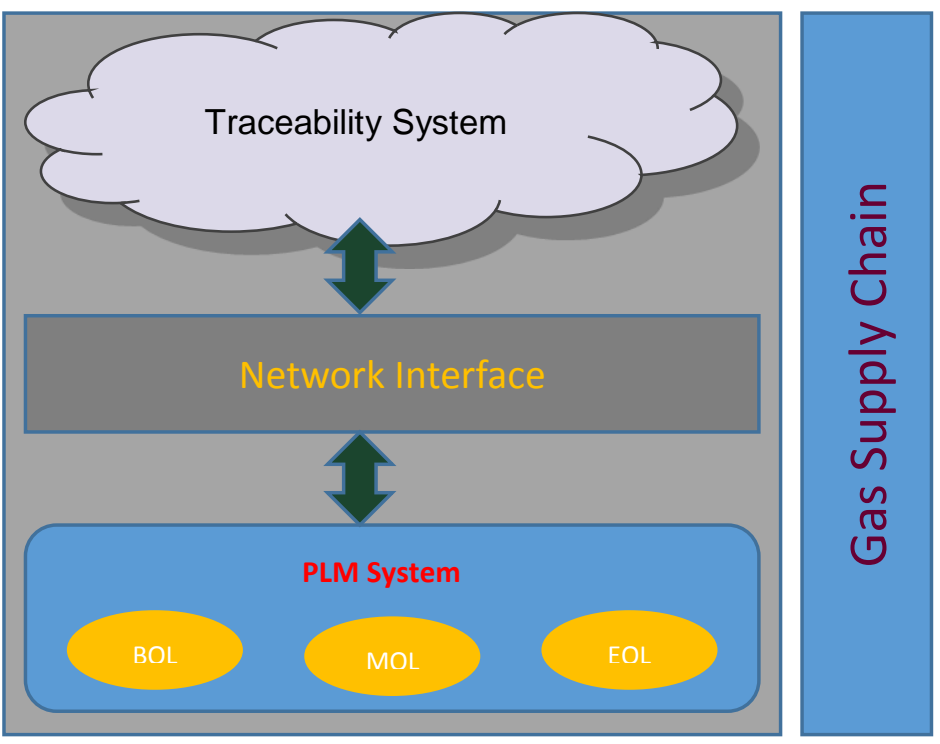

Fig. 3. Proposed Traceability System Architecture

Figure 3 shows the proposed traceability system architecture. Every cylinder has its life span. As per the conversation with concerned authorities of the company, a cylinder has 17 to 20 years life span. We use the concept of PLM (product life cycle management) to develop the system. All the required information of cylinders like date of purchase of new cylinders, date of refilling, no. of times of refilling etc. are put into the PLM system. The empty cylinders are refilled three times per month in average. The cylinders has to go through hydraulic testing to check whether there is formation of rust or not. During refilling, if the cylinders get in contact with air, rust is formed on the surface of cylinders, which detoriates the gas quality. Refilling information in the PLM system helps to check the quality issue of the cylinders when a problem is encountered.

To uniquely identify the cylinders, GS1's GTIN standard is used, which is encoded in RFID tag attached with every cylinders. RFID allows automatic identification of data encoded with it [23]. Reading and writing is done in the RFID tag 
through radio waves without physical contact of reader and tag. Active and passive are two categories of RFID tag. Active tag takes power from internal storage (battery) whereas passive tag uses power gained from radio frequency through reader. RFID works on various frequencies level: UHF (Ultra high frequency: $860 \mathrm{MHz}$ to $960 \mathrm{MHz}$ ), HF (High Frequency: $13.56 \mathrm{MHz}$ ) and LF (Low Frequency: $125 \mathrm{KHz}$ to $134 \mathrm{KHz}$ ) [23]. We use the SaaS concept of Cloud Computing and put the traceability system on to it. A web based system will be developed based on the architecture shown in figure 5, which connects the traceability system with PLM system. This system will be used by every stakeholders of the gas supply chain and disseminate all the required information when needed.

\section{Conclusion}

The on time delivery of oxygen gas in the hospitals is essential. To achieve this, the gas companies must have the proper production plan so as to manage the production as per the need of customers. A traceability system in the gas supply chain not only helps the manufacturers to properly manage the order but also it helps them to track their filled or empty cylinders in the network. In this work, a traceability framework is proposed to trace the cylinders in the gas supply chain using the concept of PLM and Cloud Computing. A trace algorithm is developed based on bi-graph model of previous work of authors. The OGTS framework proposed in the work, ensures the required exchange of information between the actors to achieve a proper traceability system. The main aim of the framework is to supply the necessary information of the cylinders when it is lost or a quality issue is encountered at any point of supply chain.

\section{References}

1. Thakur M, Hurburgh C. R: Framework for implementing traceability system in bulk grain supply chain, Journal of food Engineering, 617-626, 2009

2. Mishra D, Sekhari A, Henry S, Ouzrout Y: Traceability in product Supply chain: A Global Model, International conference on Product Life Cycle Management, University of South Carolina, Columbia, USA, 2016

3. Corina Ene: The relevance of traceability in the food chain, Economics of agriculture 2/2013, UDC: 005.6.338.439

4. Folinas D, Manikas I, Manos B: Traceability data management for food chain, British Food Journal, 108(8), 622-633, 2006

5. Regattieri A., Gamberi M., Manxini R: Traceability of food products: General framework and experimental evidence, Journal of Food Engineering 81(2007), pp 347-356.

6. W. He, N. Zhang, P. S. Tan et al: a secure RFID-based Track and Trace Solution in Supply chains, IEEE 2008. 
7. W. He, L. Tan et al: A solution for Integrated track and trace in in supply chain based on RFID and GPS , IEEE 2009.

8. R. Shougang, X. Huanliang et. al : Research on RFID-based Meat product track and traceability system, IEEE 2010.

9. R. Z. Cao, X. Y. He et. al: Establish trust from whole chain traceability, IEEE 2011.

10. C. Teng, K. Brown et. al: a service oriented Livestock management system using mobile cloud architecture, IEEE 2012.

11. N. Madhoun, F. Amine et. al: an innovative cloud based traceability architecture and service

12. I. Exposito, I. Cuinas et. al: Efficient traceability solutions in the wine production by RFID and WSN, seventh European Conference on Antennas and Propagation, IEEE 2013.

13. U. Barchetti, A. Bucciero et. al Impact of RFID, EPC and B2B on traceability management of the pharmaceutical supply chain, Dept of Innovation Engineering, University of Salento, Italy.

14. Li X., Liu X. et al: Bigraph-based modeling and tracing for the food chain system, IEEE international conference on information science and cloud computing companion, (2013)

15. Diallo T. M. L., Henry S., Ouzrout Y.: Using Unitary Traceability for an optimal product recall, Springer Publication

16. Pizzuti T., Mirabelli G.: The Global Track \& Trace System for food: General Framework and Functioning principles, Journal of food Engineering 159, 16-35, (2015)

17. Khabbazi M. R., Ismail N., Ismail Y.: Data modelling of traceability information for manufacturing control system, IEEE (2009)

18. Jansen-Vullers, C. A, Van Drop et al: Managing traceability information in manufacture, International Journal of management, 395-413, (2003)

19. R. R. Pant, G. Prakash : A framework for traceability and transparency in the Dairy Supply Chain Networks, Procedia-Social and Behaviour Sciences 189 (2015), 385394

20. M. Bevilacqua, F. E. Ciarapica et al. : Business Process Reengineering of Supply Chain and traceability System: A Case Study,Jornal of Food Engineering 93 (2009) 13-22

21. M. Thakur, Bobby J. Martens et al. : Data model to facilitate internal traceability at a grain elevator, Computer and Electronics in agriculture 75 (2011), 327-336

22. J. $\mathrm{Hu}, \mathrm{Xu} \mathrm{Zhang}$ et al : Modelling and implementation of the vegetable supply chain, Food Control 30 (2013) 341-353

23. N. EI Madhoun and F. A. Guenane: A Novel Cloud-based RFID Traceability Architecture and Service, Sorbonne Universite UPMC Univ Paris 06, UMR 7606, LIP6, F-75005, Paris, France 